\title{
Florencio Sevilla. Elogio de la edición
}

\author{
José Ramón Trujillo Martínez \\ Universidad Autónoma de Madrid
}

Quisiera yo, si fuera posible, lector amantísimo, excusarme de escribir este retrato (de rostro aguileño... las barbas de plata) por varias y animosas razones. Entre ellas, una no menor sería la reprensión que me haría el retratado, el cual habría preferido mil veces, antes que gastar el tiempo en estas páginas, dedicarlo a discutir sobre la cuenta del original en la imprenta o el centenar de errores tipográficos del Persiles. Y también sobre la calidad de uno u otro jamón ibérico. Esta punta de modestia o de honesto pudor fue característica en quien puso siempre la literatura por delante del brillo, la polémica y la promoción a costa de los clásicos. Una semblanza de Florencio Sevilla Arroyo me pide el profesor Bonilla Cerezo para la sección "Lecciones y maestros" de Creneida. Y en verdad resulta un grave aprieto, fruto del dolor de la pérdida súbita y reciente y por la amplitud de una tarea que debe aquilatarse en pocas líneas.

A pesar del descuido en los nuevos planes de estudio y del desinterés que venimos observando hacia la lectura detenida de las obras, la forma clásica de la Filología, la más genuina al decir de Erich Auerbach, continúa siendo la edición de textos. El verdadero corazón de la Filología. Florencio Sevilla (Villanueva de Guadamejud, Cuenca, 1956-Madrid, 2020) tuvo a gala ser, antes que otra cosa, un editor en el sentido más noble del concepto: interpretar los testimonios y ofrecer un texto tan accesible como fiel al original; devolver el texto a la proximidad de la mano del autor, a su intención última, sin traspasar jamás los límites de lo comprobable; entregarse al cuidado estudio de la herencia recibida y su recepción histórica. En un tiempo en que priman la cantidad y la rapidez a la hora de valorar una trayectoria, una parte central de su ministerio fue 
la del notario dedicado a la lenta compulsa de ediciones, a registrar con detenimiento todos los errores y variantes, y a fijar las lecciones de cada uno de los libros a los que prestó su atención. Se consagró a la tarea de preparar los textos antes de explicarlos en sus conferencias y trabajos, pero en primer lugar dentro del aula, con sus estudiantes de la Universidad Autónoma de Madrid (UAM), donde impartió Literatura Española Medieval y de los Siglos de Oro durante cuatro décadas.

Edoardo Sanguinetti escribió que "i classici nascono con la filologia, e sono condizionati alla filologia. Dove non si ha filologia, non si ha classico, propriamente". A poner los principales clásicos áureos y de la Edad Media al alcance de un amplio conjunto de lectores (mediante ediciones críticas, didácticas, comerciales, artísticas, facsimilares y digitales), a revaluarlos dentro del canon, dedicó el profesor Sevilla Arroyo sus mejores esfuerzos. Firme enemigo de la teorización abstracta sin base en la letra, apostó en todo momento por la edición filológica como "paso honroso", previo al análisis posterior, y como parte esencial de la comprensión de una cultura a través de sus obras mayores.

En 1970, Juan Manuel Rozas fundó el Departamento de Literatura de la UAM, que dirigiría hasta que en 1977 ganó la cátedra en la Universidad de Santiago. Durante el último tramo de este mandato, en un jovencísimo campus nacido para descongestionar - y modernizarel Madrid de la Transición, y donde se dieron cita, entre otros maestros, Fernando Lázaro Carreter, Domingo Ynduráin, Diego Catalán o Miguel Á. Pérez Priego, Florencio Sevilla se orientaría en primera instancia hacia la narrativa contemporánea, con una tesina dirigida por José Luis Varela: Aproximación a las "novelas del descontento" de Pedro de Lorenzo (1979). Su encuentro con el profesor Antonio Rey Hazas enfocó su interés por la picaresca y nuestra fecunda Edad de Oro.

Profesor ayudante y bibliotecario del Departamento de Literatura Española (1979), titular (1987), director del departamento, ya de Filología Española (1990-1994, 2003-2006) y, por fin, catedrático (2009), su trayectoria se desarrolló íntegramente en la UAM y representa a las claras la efervescencia, amplitud y repercusión que los estudios filológicos alcanzaron en ella. Siguen recordándose hoy las actividades del Seminario Internacional Edad de Oro, fundado por el profesor Pablo Jauralde, forja de varias promociones de investigadores en las salas de la 
Biblioteca Nacional de España, de notables proyectos de excelencia y de los congresos temáticos anuales, celebrados a caballo entre Cantoblanco y Cuenca. Florencio Sevilla fue codirector desde 1993 y director entre 1997 y 2012; con él —y Begońa Rodríguez como secretaria desde 2004- el seminario alcanzaría su mayor grado de internacionalización. Ya en su última década, pasó de estar dirigido a los estudiantes de literatura de la UAM a convertirse en lugar de encuentro con los de otras áreas Lengua, Historia, Arte- y universidades. No en balde, era frecuente encontrar grupos llegados desde la UCM, la Universidad de Córdoba, Ferrara o Ginebra, bajo la guía atenta de profesores como Rafael Bonilla Cerezo y Carlos Alvar.

El primer trabajo del profesor Sevilla sobre los Siglos de Oro tuvo como objeto Alonso, mozo de muchos amos (Madrid, 1624; Valladolid, 1626) de Jerónimo de Alcalá Yáńez y Ribera. Dirigida por Antonio Rey Hazas, esta tesis doctoral (1986) presentó un texto que superaba con largueza las ediciones decimonónicas, las cuales incluso llegaron a cambiar el título por El donado hablador. Lo fijó a partir del único testimonio de la Primera parte, conservado en la Hispanic Society, con una rigurosa atención textual que sería desde ese momento la pauta en sus trabajos posteriores. Le siguieron las ediciones de Lazarillo de Tormes (Plaza y Janés, 1984), Guzmán de Alfarache (Ollero y Ramos y Mondadori, 2003; Penguin Random House, 2015), el Buscón, La niña de los embustes..., hasta llegar a las veinte obras que reunió en su monumental Novela picaresca española completa (Castalia, 2001), donde rescataba en rigurosas ediciones - siempre a partir de la princeps — el más español de los géneros de ficción. Muchas de estas obras permanecían inéditas desde el Barroco o habían sufrido una lagunosa presentación: Segunda parte de Lazarillo de Tormes (1555), La picara Justina (1604), El guitón Onofre (1602), Lazarillo de Manzanares (1620), Periquillo el de las Gallineras (1662)... Dicha empresa, capital y acometida en solitario, por un lado desembocó -a través de diversos artículos y prólogos- en una reformulación y nueva propuesta de la "poética de la novela picaresca" desde el diálogo; por otro, en una meritoria función de almácigo, que ha servido como base en diversas tesis doctorales y del acicate para nuevos estudios.

Además de la picaresca, se ocuparía de otros clásicos, como la Turcarum origo (con Ana Vian, Criticón, 1989), La dama duende y Casa con dos 
puertas, mala es de guardar (con Antonio Rey Hazas, Planeta, 1989), El gran teatro del mundo (con Antonio Rey Hazas, Planeta, 1991) y, de forma muy especial, de varias ediciones didácticas o modernizadas, con actividades pensadas para el trabajo en el aula: Libro de buen amor (con Pablo Jauralde, PPU, 1988; y, ya solo, en Edelvives, 2014), La Celestina (Edelvives, 1990, 2014) e Historia de la vida del Buscón (Edelvives, 1991, 2014). Corresponden a su vivo interés por el acceso a la lectura y su preocupación por la relación de ida y vuelta entre la docencia universitaria y la Enseñanza Secundaria — nunca olvidó que buena parte de nuestros licenciados son o serán profesores de instituto y el eslabón esencial de la aproximación a los clásicos—, que lo llevaron a ser durante seis años Coordinador de COU de Literatura en Selectividad y un profesor siempre alentado por la comprensión cabal de los textos, amén de curioso de la historia de los géneros.

Es de destacar además su acercamiento temprano a la informática. En tiempos de Humanidades Digitales, resulta difícil valorar lo que tuvo de pionera su apuesta atenta a los nuevos medios de tratamiento y transmisión textual, así como la difusión que alcanzó con ello la obra de Cervantes. Fue investigador en proyectos de creación de plataformas web para el estudio del manco de Lepanto, la novela corta barroca o el erotismo en la poesía áurea. Entre otros productos, publicó con su inseparable Antonio Rey Hazas la obra completa cervantina en soporte digital — primero en disquetes (Alianza, 1996), luego en CD en Micronet (1997)_-, dirigió y nutrió la Biblioteca de Autor Miguel de Cervantes (Biblioteca Virtual Miguel de Cervantes de la Universidad de Alicante, desde 2001), promovió la Biblioteca Virtual de Humanidades de la UAM (2003) y dirigió la colección de e-books (Bolchiro, desde 2012). Por otro lado, impartió cursos de Informática y edición de textos y, desde la implantación del nuevo plan de estudios, la asignatura Edición Literaria y Nuevas Tecnologías, que tuve el gusto de compartir algunos años.

Con ser fundamental su labor como medievalista y estudioso de la picaresca, hacer memoria de la tarea del profesor Sevilla Arroyo es, antes que otra cosa, hacer historia de los últimos treinta y cinco años de los estudios cervantinos. Atraído por el legado literario de nuestro raro inventor, por su afán experimental, su finura poética, su interés por la verosimilitud alcanzada mediante un amplio abanico de recursos, 
o sencillamente por el reto que suponía abordar su obra completa, le dedicaría un ingente esfuerzo a su edición. El resultado y su presencia continuada en las mesas de novedades se antojan apabullantes. Con Antonio Rey Hazas abordó de forma sistemática la edición e introducción de todo el corpus de Cervantes: Teatro completo (Planeta, 1987; Altaya, 2005); Novelas ejemplares (2 vols., Espasa Calpe, 1991); Obra completa: vol. 1. Quijote (Centro de Estudios Cervantinos, 1993, 1994), vol. 2. La Galatea. Novelas ejemplares. Persiles y Sigismunda (CEC, 1994), vol. 3. Ocho comedias y ocho entremeses nuevos. La Numancia. El Trato de Argel. Poesía (CEC, 1995); Obra completa (18 vols., Alianza, 1996-1999); Novelas ejemplares. Selección (Espasa Calpe, 2007); Don Quijote de la Mancha (2 vols., Alianza, 1998; 2014). Volvería a editarlo en diversas ocasiones, ahora en solitario, revisado y con nuevas introducciones y notas: Obras completas (Castalia, 1999), Comedias (3 vols., Biblioteca Clásica Castalia, 2001); El trato de Argel. La Galatea (Biblioteca Clásica Castalia, 2001); El curioso impertinente. El Cautivo (Biblioteca Clásica Castalia, 2002); Don Quijote de la Mancha (Ollero y Ramos y Mondadori, 2002; Lunwerg, 2004; 2 vols., Diputación Provincial, 2005; Universidad Europea Miguel de Cervantes, 2005; Sial, 2005, con un apéndice ilustrado de José Manuel Lucía Megías); La tía fingida (Novela atribuida a Miguel de Cervantes) (Instituto Castellano y Leonés de la Lengua, 2005); Don Quijote de la Mancha (Museo Iconográfico del Quijote, 2010; Penguin Random House, 2015); La Galatea (Penguin Random House, 2016); Obras completas (4 vols., Museo Iconográfico del Quijote, 2014-2018)... Añadiré asimismo los volúmenes Cervantes. Vida y literatura (con Antonio Rey Hazas, Alianza, 1995), Cervantes, Cultura Literaria (con Carlos Alvar, CEC, 1997), la edición didáctica Don Quijote de la Mancha (con Elena Varela, Castalia Didáctica, 1997) y las traducciones del Quijote al italiano, por Patrizia Botta (Mucchi, 2015), y de las Ejemplares al inglés, por Michael J. McGrath (Cervantes \& Co., 2008), junto con un buen acervo de artículos. La mera cadencia de publicaciones no da idea fiel de la recepción, en número de ejemplares y reimpresiones, de una tarea que permitió a muchísimos lectores, especialmente los estudiantes, tener al alcance de su mano el conjunto de la obra cervantina.

En algún momento habrá que consagrar una (o varias) tesis a estudiar el impacto publicitario y también en los mass media de los 
diversos centenarios cervantinos. Los fastos del cuarto del Quijote y su extensión hasta antes y después del aniversario del fallecimiento de su autor darían además para una intriga en la que, a partes casi iguales, se mezclan la anárquica y derrochadora política cultural de nuestro país, la promoción privada y el exhibicionismo. En la receta del combinado, en el que, con varios años de antelación, se deslizaron los centenarios circuitos turísticos y gastronómicos, sin orillar las semanas culturales o "de huesos", no siempre tuvo cabida la filología, que ganó el interés de la prensa solo revestida de duelo crítico en torno a la "edición del centenario", con varias instituciones y editoriales por medio. Como el prudente Cide Hamete advirtió a su pluma, algunos levantaron la voz: “¡Tate tate, folloncicos! / De ninguno sea tocada; / porque esta impresa, buen rey, / para mí estava guardada”. Menudearon entonces, también en prensa, "titulares publicitarios" con expresiones como "el Quijote total", "el Quijote definitivo" o sencillamente "el Quijote de...".

Lo cierto es que la cuestión venía de atrás: los ańos setenta y ochenta de siglo XX habían supuesto un enorme avance en todos los frentes de los estudios literarios, pero, a pesar de las propuestas de Luis Andrés Murillo (1978), R. M. Flores (1982), John J. Allen ("A More Modest Proposal for an Obras Completas edition", 1982), Daniel Eisenberg ("On Editing Don Quixote", 1983) y Angel Basanta (1985), se carecía, en palabras de José María Casasayas (1986), de "la edición definitiva” de la obra central del canon español. Más aún: tampoco existían una Obra completa, ni una enciclopedia de materias; ni siquiera una bibliografía cervantina de veras exhaustiva. Esta última se abordó a partir del Ensayo de una Guia, del propio Casasayas, y gracias al esfuerzo posterior de José Montero Reguera. La Enciclopedia cervantina la hizo realidad Juan Bautista Avalle Arce (1997) y, convertida en Gran Enciclopedia Cervantina en quince volúmenes, la volvieron referencia indispensable Carlos Alvar y Florencio Sevilla (2002-hasta la fecha).

Si estos objetivos instrumentales devinieron realidad indiscutida, el esfuerzo por alcanzar un Cervantes crítico que superara los problemas textuales y editoriales se convertiría en la cuestión central de la crítica hispana de estos últimos veinticinco años; y en el empeño mayor de Sevilla Arroyo. La aparición de las obras completas en la editorial Alianza — a diferencia de la previa del CEC — no pasó desapercibida, 
quizá por su carácter comercial y la novedad del texto en disquetes. De ahí que los medios de comunicación (incluido El País, 13 marzo 1996) se hicieran eco y... “¡oh Belardo, aquí fue Troya!”. La historia editorial y la polémica suscitada a partir de un artículo de Francisco Rico (" "Por Hepila famosa» o cómo no editar el Quijote”, Babelia 255, El Pais, 14 septiembre 1996), que preparaba una edición del Quijote para el Instituto Cervantes, es bien conocida; por eso bastará remitir aquí al resumen de Eduardo Urbina ("Arte nuevo de editar el Quijote en este tiempo", 2000). Tras reseñar "capítulos oscuros" e intereses institucionales, concluye "que, como resultado feliz del debate, así como de las iniciativas y esfuerzos descritos, gozamos hoy de más y mejores ediciones del Quijote y de las obras completas de Cervantes, antes de dar paso a su propuesta informática superadora”.

Corría el año de 2001. En una fresca mañana de mayo, Florencio Sevilla me llamó para hablar de bibliografía material. Unos meses antes me había regalado el Cervantes completo que venía editando en Alianza, coordinado por el profesor Alvar. Habíamos charlado entonces sobre los textos incluidos en los disquetes y luego en $\mathrm{CD}$-por nuestro interés en la informática, coincidíamos en que era un paso fundamental—, pero en esta oportunidad la conversación se convirtió en un examen de lecturas, técnica habitual de evaluación en sus cursos universitarios: Julián Martín Abad, Alfred W. Pollard, Walter W. Greg, Ronald B. McKerrow, claro; pero también algunos trabajos de Jaime Moll y Mercedes Fernández Valladares, y, por supuesto, A New Introduction to Bibliography de Philip Gaskell, que había leído en la edición de 1995 en la BNE y acababa de aparecer vertido al español en una traducción revisada por José Martínez de Sousa. A la sazón, mi familia tenía una imprenta, yo regía una incipiente editorial y a Florencio Sevilla siempre le parecieron fascinantes las novedades que le iba contando, como los efectos de combinar QuarkXPress con la LaserWriter para los juegos de pruebas y la llegada del CtP, que acababa de eliminar los procesos de imposición de fotolitos sobre astralones, insolación y revelado de planchas, y también la corrección sobre diazos.

Un mes después me encontraba en su casa, donde estuvimos contando caracteres y blancos del Quijote por líneas, por planas, por cuadernos... Las líneas por plana, los tipos sólidos totales, la disposición de epígrafes, poemas, cartas... Contaba yo y escrutaba él mis resultados, toda vez que 
ya había hecho cien veces la cuenta y tenía incluso simulaciones de las planas en el ordenador. No pasó la cosa de un par de sesiones, pero el interés por las consecuencias textuales de los procesos de la imprenta de dos golpes pronto se iba a convertir en la siguiente liza.

Algunos de estos datos, actualizados y arropados metodológicamente, vieron la luz años después en forma de capítulos ("La "cuenta del original» en el primer Quijote: consecuencias textuales"; " "Cuenta del original» y remedios de cajista en la princeps del primer Quijote"; "Madrid, Juan de la Cuesta, 1605 y 1615: el Quijote "definitivo"”; "El texto definitivo del Quijote, según Cervantes"; " "Famoso [y valiente] hidalgo»: sobre conjeturas y deturpaciones textuales"; "La "cuenta del original» a la luz de la "cuenta del impreso»"; "La intervención de la imprenta en los tres primeros Quijotes de Juan de la Cuesta”; o "La intervención de la imprenta en el texto del tercer Quijote de Cuesta (1608)"), y sería deseable que algún día se recogieran en un volumen.

El Bibliographical turn se produjo durante el centenario de la impresión de la Primera parte y tras la inusitada atención crítica que suscitó la coincidencia de la tesis Manuscritos e imprenta (2005) de Sonia Garza; Aqui se imprimen libros. La imprenta en la época del Quijote, de José Manuel Lucía Megías (2005); Filologia dei testi a stampa (Area Iberica), de Patrizia Botta (ed.) (publicado en 2005, aunque el seminario tuvo lugar en Pescara, en noviembre de 2003), la Azione Integrate ItaliaSpagna "Filologia dei Testi"; y sobre todo El texto del "Quijote" (2006) de Francisco Rico. La década pasó entre "yerros" y duendes de imprenta y "remedios" de cajistas. Es lugar común que la espada se forja a martillazos. La competición editorial por el Quijote ha dejado además un buen número gavilla de artículos, ensayos y reseñas llenos de interés, de ambición y de saña, cuyas páginas aguardan ya a su historiador. Entre otras felices consecuencias, el profesor Sevilla dedicó a la "Imprenta manual y edición de textos áureos" el XXVIII Seminario Edad de Oro, donde se dieron cita algunos de los principales especialistas y se expuso con detalle al numeroso público la tecnología de impresión en tiempos de Cervantes. Sin embargo, la atención dedicada y los magros resultados inobjetables lo habían llevado a reafirmarse en varias certezas: 1) el descuido material de la edición de 1605 y la amplia panoplia de recursos de los componedores; 2) que la bibliografía textual es solo una herramienta más y aporta poco 
en los casos analizados; 3) que es preferible el testimonio abrigado por una recepción histórica que las conjeturas sin base.

Al reflexionar Casasayas (1986) sobre qué tipo de edición debía considerarse definitiva, había propuesto como guía el respeto de los testimonios, advirtiendo con antelación a las polémicas que "puede ocurrir que algún día se descubra que Cervantes quiso realmente decir «por Epila famosa» con [algún sentido] que ahora desconocemos con exactitud, y entonces todas las enmiendas ("por hacerla famosa») se vendrían abajo”. Si, como afirma reiteradamente Trapiello, en la obra y la vida de Cervantes todo parece articulado con la bisagra de las conjeturas, entonces las principes y la documentación supérstite deben ser el fulcro sobre el que apoyar todo acercamiento. Florencio Sevilla convirtió en realidad crítica las viejas advertencias de Bédier sobre la peligrosa creación de monstruos compuestos y las recientes de Ángel Rosenblat acerca del necesario respeto a los textos y su opinión contra las enmiendas injustificadas de los «correctistas».

Con motivo de la aparición de una edición actualizada de las Obras completas de Cervantes, publicada por el Museo Iconográfico del Quijote de Guanajuato (4 vols., 2010-2016), en una entrevista sintetizó su conocida apuesta y la urgencia de seguir actualizando su aparato crítico; bien oiréis lo que dirá:

los criterios textuales aplicados a la totalidad de las obras cervantinas responden a una vieja convicción crítica que vengo ratificando desde hace ya unos veinticinco años y consolidando cada día con más solidez, según he expuesto en múltiples sitios: la necesidad imperiosa de respetar a ultranza a los únicos testimonios autorizados de los textos cervantinos, las ediciones príncipe, con tal de preservar la legitimidad del texto ofrecido al lector por encima de la incertidumbre de la más aguda divinatio, que suele acabar deturpando la letra original de Cervantes... Esto es, nuestra nueva edición sigue empeñada en depurar permanentemente las lecturas de los únicos testimonios originales, convencidos de que cualquier intervención crítica en los mismos - al margen, claro está, de la corrección de erratas evidentes - supone siempre, en tanto que no documentada y no documentable, un salto al vacío con tantas posibilidades de error como de acierto, lo que impone el respeto esencial a los ori- 
ginales conservados como única opción ecdótica responsable $\left(\mathrm{M}^{\mathrm{a}}\right.$ Augusta da Costa "Entrevista con Florencio Sevilla Arroyo», Caracol 1, 6 (2013), pp. 234-249).

El profesor Sevilla no deja solo una vasta tarea como editor y crítico, sino una prolongada actividad como formador de especialistas: al margen de las disputas y discrepancias, nos lega su inconformismo y la lección de una técnica honesta y sistemática a la hora de abordar las obras: frente a la asunción de lo reputado, el interrogatorio minucioso de los textos; frente a la mera abstracción teórica, el recurso a las pruebas y testimonios; frente a la tentación de la bombonera, la unicidad del esfuerzo y el conocimiento en profundidad de todas las obras de un autor y de un género; frente a la édition savante y la afectación erudita, el texto crítico y la accesibilidad. Muchos son los caminos de la filología. Tuvo a gala ser, antes que cualquier otra cosa, un editor de textos en el sentido más noble: aquel que devuelve el texto a la voluntad original de su autor y nos lo entrega para disfrutarlo sin apenas escollos. Obras nacidas del ingenio, que ganan lectores gracias a su esfuerzo silencioso y van creciendo en los brazos de la estampa. 